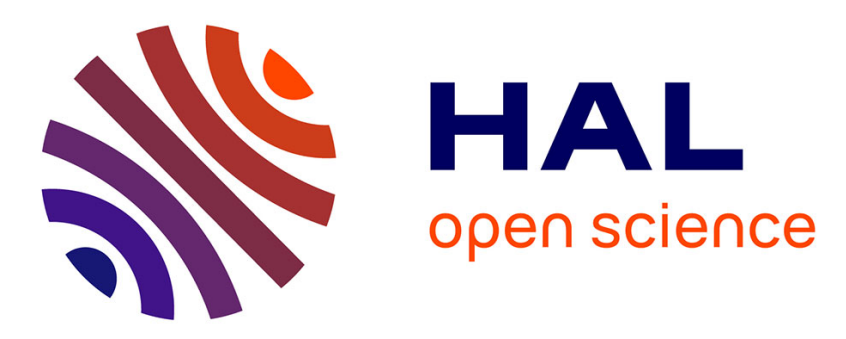

\title{
Analysis of strain and disordering kinetics based on combined RBS-channeling and X-ray diffraction atomic-scale modelling
}

Xin Jin, Alexandre Boulle, Alain Chartier, Jean-Paul Crocombette, Aurelien

Debelle

\section{To cite this version:}

Xin Jin, Alexandre Boulle, Alain Chartier, Jean-Paul Crocombette, Aurelien Debelle. Analysis of strain and disordering kinetics based on combined RBS-channeling and X-ray diffraction atomic-scale modelling. Acta Materialia, 2020, 201, pp.63-71. 10.1016/j.actamat.2020.09.076 . hal-03034474

\section{HAL Id: hal-03034474 https://hal.science/hal-03034474}

Submitted on 2 Dec 2020

HAL is a multi-disciplinary open access archive for the deposit and dissemination of scientific research documents, whether they are published or not. The documents may come from teaching and research institutions in France or abroad, or from public or private research centers.
L'archive ouverte pluridisciplinaire HAL, est destinée au dépôt et à la diffusion de documents scientifiques de niveau recherche, publiés ou non, émanant des établissements d'enseignement et de recherche français ou étrangers, des laboratoires publics ou privés. 


\title{
Analysis of strain and disordering kinetics based on combined RBS-channeling and X-ray diffraction atomic-scale modelling
}

\author{
Xin Jin ${ }^{1,2, *}$, Alexandre Boulle ${ }^{2}$, Alain Chartier ${ }^{3}$, Jean-Paul Crocombette ${ }^{4}$, \\ and Aurélien Debelle ${ }^{1,4,{ }^{* *}}$ \\ ${ }^{1}$ Université Paris-Saclay, CNRS/IN2P3, IJCLab, 91405 Orsay, France \\ ${ }^{2}$ Institut de Recherche sur les Céramiques (IRCer), CNRS UMR 7315, Université de \\ Limoges, Centre Européen de la Céramique,12 rue Atlantis, 87068 Limoges, France \\ ${ }^{3}$ Université Paris-Saclay, CEA, Service de la Corrosion et du Comportement des \\ Matériaux dans leur Environnement, 91191 Gif-sur-Yvette, France \\ ${ }^{4}$ Université Paris-Saclay, CEA, Service de Recherche de Métallurgie Physique, 91191 \\ Gif-sur-Yvette, France \\ ${ }^{*}$ Corresponding author: xin.tlg.jin@outlook.com \\ ** Corresponding author: aurelien.debelle@ijclab.in2p3.fr
}

\begin{abstract}
Ion beams delivered by particle accelerators are routinely used to emulate harsh, radiative environments and they also constitute the foundations of the modern microelectronics industry. To characterize irradiated materials, numerous experimental and computational techniques can be implemented, but it is extremely difficult to effectively intertwine them, and to compare the associated data. In the present work, we present an integrated, experimental and computational approach that uses a same set of molecular dynamics simulations to generate signals of Rutherford backscattering spectrometry in channelling condition and $\mathrm{X}$-ray diffraction, with $\mathrm{UO}_{2}$ as a test-case material. From these signals, parameters to monitor the damage level are computed, compared and confronted with experimental data. Although the evolution of the strain and disordering kinetics obtained by simulations differ on an absolute scale from those obtained experimentally (a discrepancy inherent to the method used to generate the atomic-scale data), a very good relative agreement is obtained, which demonstrates the validity of the approach, hence providing a new tool for the fine study of irradiation effects in materials.
\end{abstract}

Keywords: Irradiation effect; RBS/C; X-ray diffraction (XRD); Molecular dynamics simulations; Defects 


\section{Introduction}

In our modern society, there is an increasing number of activities involving the use of energetic ions delivered by particle accelerators, which encompass (but are not limited to) health (with hadrontherapy) [1], high-energy and nuclear physics [2, 3], astronomy [4], and materials science. In this latter domain, several industries and research fields have benefited greatly from the ion irradiation process which can result in both the controlled introduction of foreign atoms in the lattice materials and the tailored modifications of the atomic structure. Ion doping of semiconductors, for instance, represents one vital step for modern integrated-circuit manufacturing [5, 6], and it is considered as a promising route for developing materials with applications in novel optoelectronic devices [7] as well as in spintronics [8] and nanophotonics [9]. In a broader framework, ion irradiation is explored to tailor various properties of nanomaterials including mechanical, electrical and magnetic properties [10-12]. Ion irradiation can also be used to shape materials down to the nanoscale [13-15]. In the field of nuclear energy, ion irradiation is used worldwide as a proxy to neutron irradiation for testing and qualifying nuclear materials [16-18].

Ion irradiation clearly represents a powerful tool in materials science because it allows driving the materials in out-of-equilibrium conditions. This process has a large number of advantages that span from the possibility to perfectly control the nature and the quantity (also called fluence, i.e. (ion) $/ \mathrm{cm}^{2}$ ) of the ions, the flux and energy of the beam, to applicability to nearly all types of materials. However, it has its drawbacks, the main one being the quasi-systematic disordering of the perfect stacking of the lattice atoms because of the permanent displacement of these latter during the energy deposition process. Indeed, if the energy transferred to target atoms is larger than a threshold displacement energy, the primary knock-on atom is ejected, sometimes with a velocity sufficient to induce a subsequent collective displacements of target atoms; this phenomenon is referred to as a collision cascade [19]. After this collisional stage, an energy dissipation phase occurs, followed by a diffusional phase, and defect recombination may occur. At the end of this multi-step process, the target atoms may remain displaced, inducing a residual disorder. Increasing the irradiation fluence usually leads to defect clustering and formation of extended defects or even amorphous clusters [20]. As a consequence, the irradiation process can result in macroscopic changes of the material properties that include mechanical [21], thermal [22], and chemical properties (e.g., dissolution rate [23]), as well as frequent significant volume change $[24,25]$. Therefore, it is of crucial importance to have a comprehensive understanding of the basic mechanisms underlying defect formation, accumulation and reorganization during ion irradiation. For this purpose, advanced tools for material characterization and modelling are required.

Investigating irradiation effects in materials has fueled a huge amount of experimental and computational studies over the last decades. Experimentally, transmission electron microscopy (TEM) is an invaluable tool for direct defect imaging. Techniques such as Raman spectroscopy, positron annihilation spectroscopy, Rutherford backscattering spectrometry in channeling mode (RBS/C), and X-ray diffraction (XRD) are widely used to evaluate the disorder level in irradiated materials (see ref. [26] and references therein). Most of the time, they rely on a phenomenological approach where disorder is quantified through a simple parameter such as the weakening of the Raman lines, the fraction of positron annihilations 
with core or shell electrons, the backscattering yield in RBS/C, or the intensity attenuation in XRD. Simulation methods for modelling irradiation effects encompass a vast range of time and length scales, from the femtoseconds to centuries, from the atomic level to macroscopic dimensions (see refs. [27, 28] for reviews). Most widely used methods are molecular dynamics (MD) for the study of primary defects, kinetic Monte-Carlo (KMC) that addresses the defect migration, dislocation dynamics to handle complex extended defects, and finite element modelling (FEM) for predicting the elastoplastic response of large systems. Note that these techniques can be parameterized using quantum-mechanical calculations. Ideally, experiments and simulations are intertwined, which allows validating computational techniques and interpreting experimental data. Another step towards an integrated experimental and computational approach lies in the generation of experimental-like signals using computational data as input data. This approach provides an efficient way to establish direct connections between actual defective structures (hardly accessible by experiments) and phenomenological parameters (derived from experiments).

In the present paper, we propose such an integrated approach. MD defective cells, used to mimic irradiated materials, serve as input data to dedicated codes to produce RBS/C and XRD signals. Uranium dioxide, $\mathrm{UO}_{2}$, has been chosen as a test-case material, as it is the most widely used nuclear fuel worldwide and has thus received a considerable attention during the past 50 decades. A recent review of irradiation effects in $\mathrm{UO}_{2}$ can be found in, e.g., ref. [29]. The well documented behavior of this material under irradiation, as well as the substantial quantity of corresponding data (including our own data), were two major reasons to select this material to implement our integrated approach. We show here that $\mathrm{RBS} / \mathrm{C}$ and XRD signals can be generated from a same set of MD data and used to compare the computed results with experimental characterizations.

\section{Simulation and analysis methods}

\subsection{MD simulations and defect analysis methods}

Radiation damage was reproduced with the Frenkel pair (FP) accumulation method which has the advantage of bypassing the collision cascade stage and directly simulates the evolution of defects by continuously introducing FP or by reaching a final concentration of FP at once, resulting in considerably shorter computing times [30, 31]. It has proven efficient to study the complete disordering processes in $\mathrm{Ni}$ and Ni-based alloys [32], in $\mathrm{UO}_{2}$ [33], but also the amorphization processes in lanthanum pyrozirconate [30] and $\mathrm{SiC}$ [34], and the complex defects in irradiated Fe [35].

The cells used in the MD simulations have a cubic shape. The side length of a pristine one is $21.6 \mathrm{~nm}$. Each cell contains 768000 atoms. The $\mathrm{z}$ direction of the MD cell is along the [001] direction of a $\mathrm{UO}_{2}$ unit-cell. The nominal disorder of the cell is defined as the displacements (of uranium atom) per uranium atom (dpU, similar to the displacements per target atoms (dpa) but referring only to uranium atoms) which ranges here from 0.01 to 7.85. More details about the MD simulations can be found in ref. [33]. The conversion of 
dpa to dpU can be calculated as follows:

$$
d p U=d p a \frac{N_{t}}{N_{U}} \frac{d_{U}}{d_{t}}
$$

in which $N_{t}$ is the total target atom number, $N_{U}$ is the total Uranium atom number, $d_{t}$ is the displacements of total atoms at a certain position and $d_{U}$ is the displacements of Uranium atoms at the same position. According to SRIM Full-Cascade calculations [36], the displacement threshold energies, $E_{d}$, for $U$ and for $O$ can significantly affect the ratio of $d_{U}$ over $d_{t}$. When $E_{d}(U)$ equals to $40 \mathrm{eV}$ and $E_{d}(O)$ equals to $20 \mathrm{eV}$ [37], for experimental data used at Section 3 (20 keV He ions, $500 \mathrm{keV}$ La ions and $500 \mathrm{keV}$ Ce on $\mathrm{UO}_{2}$ ), the ratios of $d_{U}$ over $d_{t}$ are similar, which are around 0.253 at the position of maximum damage. So $1 \mathrm{dpa}$ is equivalent to $0.760 \mathrm{dpU}$ at that position.

We must highlight that there is a true difference between, on the one hand, the dpU level obtained in the current MD cells produced by the FP accumulation method (MD-dpU) and, on the other hand, the dpU level derived from the SRIM Full-Cascade calculations (SRIM-dpU) which we performed in the framework of the irradiation experiments. It is known that SRIM simulations (using the Kinchin-Pease model or the Full-Cascade option, see ref.[38]) overestimate the actual number of stable defects. Indeed, they rather provide the total number of displaced atoms without considering any physical mechanism for a potential recombination, as it occurs, based on MD simulations, during the thermal spike phase of collision cascades [39]. In addition, all the FPs introduced in the MD cells, in the current work, do contribute to defect evolutions, whereas even part of the stable defects surviving at the end of the collision cascade may further recombine or annihilate due to thermal migration. Hence, we cannot compare directly MD-dpU and SRIM-dpU levels, and we can expect onsets of defect changes to take place at MD-dpU lower than SRIM-dpU. These remarks will be used in the following to explain the difference between computed and experimental strain and disordering kinetics.

The OVITO code [40] was used to visualize and analyze the MD cells. Concentrations of point defects, namely interstitials (I) and vacancies (V), were derived from a Voronoi analysis method [41]. Dislocation types and densities were determined by the dislocation analysis (DXA) method [42, 43]. Fig.1(a) presents the visualization of dislocations in damaged MD cells, in which $1 / 3<111>$ Frank dislocations (blue) are clearly in the form of loops, whereas it is difficult to distinguish $1 / 2<110>$ perfect loops and lines (green).

\section{$2.2 \mathrm{RBS} / \mathrm{C}$ simulations and analysis}

RBS/C simulations were performed with a recently developed Monte-Carlo code called RBSADEC which stands for Rutherford Backscattering Simulation in Arbitrary Defective Crystals [44]. This code was subsequently improved by us in order to extend its applicability to more simulation conditions [45]. The code is based on the Binary Collision Approximation (BCA) and can simulate RBS/C spectra from targets containing arbitrary atomic structures. The trajectories of probing ions are determined by a search-and-collide process taking into account actual target crystalline structures [46], while an algorithm describing the interaction between ions and amorphous targets is used to calculate the trajectories of backscattered ions in order to accelerate the simulations. 
For the current simulations, the probing ion was defined as a $3.085 \mathrm{MeV}$ He ion (corresponding to most available experiments). The detector energy resolution was fixed at 15 $\mathrm{keV}$. The one-dimensional root mean square ( $\mathrm{rms}$ ) thermal vibration magnitudes for $\mathrm{U}$ and O atoms were set to $6.5 \mathrm{pm}$ and $9 \mathrm{pm}$ respectively [47]. For each simulation, periodic boundary conditions were applied to the $\mathrm{x}$ and $\mathrm{y}$ directions of the MD cells, while the projectile entered the target along the $\mathrm{z}$ direction. Thus, the simulated target can be regarded as a thin layer with a $22 \mathrm{~nm}$ thickness. Three sets of simulations were performed. For each set, before applying the periodic boundary conditions, the cells were rotated in order to have the $\mathrm{z}$ direction along, alternatively, the [001], [010] and [100] directions. The purpose was to detect a potential anisotropy of material response to defect formation. In addition, a simulation in a random configuration was also performed for each cell in order to have the maximum backscattering yield as a normalization criterion. Considering that the total number of the cells was high (897 cells), a TRIM-based amorphous slowdown approach was used, which allowed a quicker calculation in RBSADEC, see ref. [? ] for more details.

Fig.1(b) depicts several examples of RBS/C spectra generated with RBSADEC using $\mathrm{MD}$ cells of different dpU levels. Blue lines represent the RBS/C spectra of $3.085 \mathrm{MeV} \mathrm{He}$ ions on $\mathrm{UO}_{2}$ in a random configuration, and orange lines represent the $\mathrm{RBS} / \mathrm{C}$ spectra along the $<001>$ aligned direction. Unlike usual experimental spectra measured for bulk targets, the spectra in Fig.1(b) are peak-shaped, since the target thickness is very small $(\sim 22 \mathrm{~nm})$. In this case, it is reasonable to assume that, essentially, direct scattering events contribute to the yield, and the dechanneling component can be neglected. This assumption facilitates the determination of the (RBS/C) disorder fraction, $f_{d}$, in each of the MD cells, which can be calculated as follows:

$$
f_{d}=\frac{y_{a}-y_{p}}{y_{r}-y_{p}}
$$

in which $y_{r}$ represents the total yield of a random spectrum, $y_{a}$ represents the total yield of an aligned spectrum for a defective cell, and $y_{p}$ represents the total yield of an aligned spectrum generated from a pristine sample.

\subsection{XRD simulations and analysis}

Similar to the RBS/C simulations, the MD cells were used to generate XRD signals and the three [001], [010] and [100] directions have been considered. For each orientation, two sets of calculations have been performed with the $\mathrm{x}$ direction of the MD cell parallel to two different directions of the unit-cell, and the corresponding strain and disorder were averaged. For instance, for a [001] orientation of $\mathrm{UO}_{2}$, the [100] and [010] directions are successively set parallel to the $\mathrm{x}$ direction. For each data set, the three-dimensional (3D) amplitude distribution, $A$, was calculated in the vicinity of the 004 Bragg reflection using the following equation [48]:

$$
A=\sum_{j} f_{j}(\mathbf{S}) \exp \left(2 \pi i \mathbf{S} \cdot \mathbf{r}_{j}\right)
$$

where $\mathbf{S}$ is the scattering vector, $f_{j}$ is the atomic scattering factor of atom $j, \mathbf{r}_{j}$ is the position of atom $j$. Since in the disordered cell the atoms are not regularly distributed on a 3D lattice, fast Fourier transform algorithms can not be used. Instead we used a direct 
summation approach accelerated by the use of a general purpose graphical processing unit (GPU).

The 3D amplitude distribution is converted to a 2D intensity distribution (i.e., a Reciprocal Space Map, RSM) with [49]:

$$
I\left(S_{x}, S_{z}\right)=\int d S_{y} \cdot A(\mathbf{S}) A^{*}(\mathbf{S})
$$

where the $S_{i}$ represents the component of $\mathbf{S}$ along the direction indicated by the subscript $i$. Complete details concerning this approach will be given in a separate article. For the sake of the present article, it is sufficient to mention that this approach allows to derive the lattice strain and the disorder parameter (the Debye-Waller (DW) factor) for all orientations of the MD cells. Examples of 004 RSMs of $\mathrm{UO}_{2} \mathrm{MD}$ cells for increasing damage levels are given in Fig.1(c). These RSMs display the 2D intensity distribution in a $(H, L)$ reference frame where $H$ and $L$ are the coordinates of the scattering vector [50]:

$$
\mathbf{S}(\mathbf{H}, \mathbf{K}, \mathbf{L})=H \mathbf{a}^{*}+K \mathbf{b}^{*}+L \mathbf{c}^{*}
$$

where $\mathbf{a}^{*}, \mathbf{b}^{*}$ and $\mathbf{c}^{*}$ are the reciprocal space basis vectors.

The inspection of Fig.1(c) reveals several features. The RSM from the pristine cell correspond to the theoretical signals of a perfect crystal, i.e., a $2 \mathrm{D}$ Laue function. For increasing fluence, we observe both a shift of reciprocal lattice point towards lower $L$ values, and a spread of the intensity out of the specular row (characterized by $H=0$ ). The former is indicative of the development of tensile strain, $e$, within the MD cell, which can be directly quantified through [50]:

$$
e=\frac{L_{0}-L}{L_{0}}
$$

where $L_{0}$ is the $L$ value of the Bragg peak in a pristine sample (here $L_{0}=4$ ). The weakening of the specular intensity is quantified by the DW factor and can be estimated from the RSMs via:

$$
D W=\sqrt{\frac{I^{H_{\max }}}{I_{0}^{H_{\max }}}}
$$

in which $I^{H_{\max }}$ is the intensity integrated along the row $H=H_{\max }$ where the spot of the maximum intensity is located, and the subscripts 0 refers to the pristine material for which $H_{\max }=0$. The disorder fraction obtained from the XRD simulations is defined as $1-D W$.

\section{Results}

\subsection{Defect evolution sequence}

Let us first consider the defect evolution obtained by a direct analysis of the MD cells in which radiation damage was mimicked by the Frenkel pair (FP) accumulation method. Fig.2(a) gives the point defect concentration and Fig.2(b) gives the dislocation densities expressed in $\mathrm{m}^{-2}$ (which corresponds to the total dislocation length of a given type, divided by the cell volume) as a function of irradiation dose - dpU. 
The defect evolution follows a sequence that has been previously identified, and, overall, confirmed by TEM analysis [33]. The sequence can be decomposed as follows. Below $0.09 \mathrm{dpU}$, point defects are predominant, and the dislocation density is very low. From 0.09 dpU, $1 / 3<111>$ Frank dislocation loops form and constitute the main defect type up to 0.25 dpU. Formation of these dislocation loops is accompanied with a decrease in the interstitial concentration, which strongly suggests that the loops are of interstitial type. At around $0.12 \mathrm{dpU}, 1 / 6<112>$ Shockley partial dislocations are detected, but they rapidly react with Frank loops to form $1 / 2<110>$ perfect dislocations [51]. These latter initially form as loops, but gradually coalesce and transform into lines. Note that, unlike Frank loops which are easily distinguishable among other defects, perfect loops and lines can be hardly separated. Above $1 \mathrm{dpU}, 1 / 2<110>$ perfect dislocation lines are predominant over the other types of dislocations. At the same time, the point defect concentration reaches a steady state, with vacancies found in a much larger concentration than interstitials. These results indicate that at this stage, interstitial point defects are efficiently trapped at dislocation lines. This general sequence of defect evolution is in accordance with recent combined experimental and computational results [33, 52-54]. A question that now arises is how this sequence of transformations affect the XRD and RBS/C signals, and what can we learn from the latter?

\subsection{Disordering kinetics}

In order to compare the results of the simulations of the two techniques, we first plotted disordering kinetics using the disorder parameters obtained by XRD and RBS/C. Fig.3(a) shows the disorder obtained from XRD, while Fig.3(b) shows that derived from RBS/C; results along the [001], [010] and [100] directions of the $\mathrm{UO}_{2}$ unit-cell are presented. In inset of the figures, the abscissa is in linear scale. The two disordering kinetics exhibit a very similar shape, which means that the two techniques could be used interchangeably to monitor the disordering process in irradiated materials. From a practical standpoint, this is an important result because, although RBS/C is to date more popular to evaluate the disorder in irradiated materials, XRD is more easily accessible and this could facilitate the characterization.

Specificities of the techniques in terms of defect sensitivity are given in the following. The absolute values of disorder obtained by XRD and RBS/C differ; this is expected as both techniques rely on very different physical phenomena, namely the interference of scattered Xrays on the one hand and the backscattering of energetic ions on the other hand. Moreover, the disorder measured by XRD is dependent on the considered $H K L$ reflection since the Debye-Waller factor, which is used to quantify the disorder [55], is a function of the scattering vector $\mathbf{S}(\mathbf{H}, \mathbf{K}, \mathbf{L})$, while the backscattering yield changes with the probing ion energy.

Nonetheless, the relative evolution of both disorders remains perfectly comparable. Both kinetics show common features that can be related to the stages of the sequence of defect evolution above-described (a quantitative support for this correlation is given in the Discussion part). First, the disorder grows at a relatively low rate with increasing dpU when, principally, point defects are present. Then, when dislocation loops form, the disordering rate considerably increases and the disorder reaches a maximum value at around $0.4 \mathrm{dpU}$. Finally, after this maximum, the disorder starts to decrease (at least with the current evalua- 
tion tools), which can be related to the presence of, essentially, dislocation lines surrounding defect-depleted regions.

It should be noted that at high dpU, for both techniques, the disorder in the different directions start to diverge and exhibit seemingly random fluctuations. Presumably, these features could be due to the so-called polygonization process, in which dislocations pile-up and lead to the formation of sub-crystallites with slightly different orientations $[56,57]$. Such resulting boundaries can act as efficient defect sinks. An in-depth analysis of the MD cells (not shown here) indeed indicates that, depending on the orientation of the cell, 2 to 3 rotated sub-crystallites co-exist within the MD cells, i.e., at high fluences the structure of the cells differs depending on the direction. It can be expected that in larger cells, such divergence would statistically disappear. A detailed investigation of that point will be given in a forthcoming paper.

Fig.3(c) presents the disordering kinetics obtained from RBS/C experiments on $U_{2}$ irradiated with $500 \mathrm{keV}$ La ions at room temperature (see the supplemental material in ref. [58]). The displacements per target atoms (dpa) obtained from SRIM Full Damage cascade (FD) calculations [36] were converted into dpU. Qualitatively, the curve from the RBS/C simulations and the experimental one exhibit very similar trends, and even the apparent decrease in disorder at high $\mathrm{dpU}$ is reproduced.

Quantitatively, there are some differences we address hereafter. Compared with the simulation results, the maximum disorder in the experiment is almost 4 times lower. As explained in ref. [33], this is because the dislocation density in the MD simulations is much higher than that in the experiments due to a very high damage rate, $K_{0}$, in the simulations. According to SRIM calculations, the experimental dose rate is around $3 \times 10^{-3} \mathrm{dpU} / \mathrm{s}$ at the damage peak, while it is $1.5625 \times 10^{9} \mathrm{dpU} / \mathrm{s}$ for the MD simulations. Assuming that the dislocation density is proportional to $K_{0}^{1 / 6}$ [59], the dislocation density in the MD cells is thus around 100 times higher than that in the real samples.

Another difference is that it takes around 20 times more dpU to reach the maximum level in the experiments. The first reason for this is, obviously, the higher defect densities resulting from the higher damage rate in the simulations. Thus, defect transformations can occur earlier in the MD cells. The second reason is due to the very definition of SRIM-dpU and MD-dpU as mentioned in Sec.2.1. The former corresponds to displaced atoms calculated in SRIM, whereas the latter is related to the actual number of defects introduced in the MD cells, which fully contribute to the evolution of defects. It is known that the SRIM results tend to overestimate the actual defect numbers, i.e., the remaining displacements after potential recombination [60, 61].

Nonetheless, and above all, it is important to emphasize that the computed and experimental disordering kinetics agree qualitatively, which represents a notable result because it demonstrates the relevance of our procedure and in the same time, it provides a support to previous interpretations of experimental data that were based on phenomenological descriptions. Furthermore, considering the good agreement between the computed RBS/C and XRD data, we can conclude that the whole integrated approach is valid. A very similar disordering kinetics has been observed in other materials including cubic yttria-stabilized 
zirconia (YSZ) [62, 63], MgO [64], Ni [65] and Ni-based equiatomic binary alloys [66]. One common feature of these materials is that either the entire crystal or the cationic sub-lattice has a face-centered-cubic (FCC) structure, indicating the possibility of having similar defect evolutions (as already partly shown). Therefore, the present qualitative description of the defect transformation sequence should hold for these materials as well, and this conclusion can be based on experimental data only (there is no need to perform MD calculations).

\subsection{Elastic strain kinetics}

In addition to disorder, XRD permits the determination of the elastic strain, which can be considered as another quantity to monitor the radiation damage. Fig.4(a) shows the irradiation-induced elastic strain kinetics derived from the XRD simulations using MD cells as input data. As for RBS/C, the [001], [010] and [100] directions were studied. Similar to the disorder, the strain kinetics displays distinct features that can be ascribed to a given type of defects (a quantitative support for this correlation is given in the Discussion part as well). When point defects are dominant, the elastic strain buildup is fast. When dislocation loops dominate, the strain keeps on increasing (with a slightly lower rate), to reach a maximum of $1.9 \%$ at around $0.28 \mathrm{dpU}$. From this point, the strain is partially relieved, which can essentially be related to perfect dislocations being the dominant defects. A slightly anisotropic evolution is observed in the three crystallographic directions, similarly to what is observed in the disordering kinetics. It is also worth noting that the maximum of the elastic strain is reached at a lower $\mathrm{dpU}$ value as compared to that of the disorder $(0.28$ instead of $0.4 \mathrm{dpU}$ ); we will comment this difference hereafter.

Fig.4(b) and Fig.4(c) show the elastic strain kinetics from $\mathrm{UO}_{2}$ irradiated with $20 \mathrm{keV}$ $\mathrm{He}$ ions and $500 \mathrm{keV}$ Ce ions, respectively. As for the disorder addressed earlier (with La ions, which are equivalent to $\mathrm{Ce}$ ), the computed strain kinetics exhibit a qualitative agreement with the experiments. Due to the very high dislocation density in the MD cells, the maximum strain computed from the simulations is around 3 to 4 times higher than that in the experiments. Regarding the delay in reaching the maximum level, since Ce and La are similar in terms of mass and solubility in $\mathrm{UO}_{2}$, a same explanation as for the disordering kinetics holds for the strain. In contrast, the strain kinetics from $20 \mathrm{keV}$ He irradiations is shifted towards higher dpU values by only a factor of 6 (instead of 20 for the disorder). As shown in ref. [61], the discrepancy between BCA models and MD calculations intensifies with increasing the primary knock-on atoms (PKA) energy, with much less surviving defects in MD (than for BCA) at high energy. In the current case, the median PKA energy of 500 $\mathrm{keV} \mathrm{Ce}$ ions is obviously larger than that of $20 \mathrm{keV}$ He ions. Therefore, it is expected that the difference between SRIM-dpU and MD-dpU is smaller for He than for Ce irradiations, explaining why the delay is reduced for the He irradiation experiment.

\section{Discussion}

From the previous section, it can be concluded that strain and disorder are remarkably correlated with the defect densities derived from the quantitative defect analysis of the MD cells. On the one hand, strain develops as soon as point defects are formed, and keeps increasing when interstitial defects agglomerate into Frank dislocation loops. When the 
concentration of Frank loops decreases, the strain decreases as well. On the other hand, disorder is more related to the presence of dislocations than point defects. To go beyond these simple qualitative observations, we propose hereafter to decompose the computed strain and disordering kinetics into components associated with the concentration of each type of defects. We averaged the results obtained along the three main directions to smooth out the observed fluctuations.

Let us start with the strain. The elastic strain kinetics is fitted assuming a linear relationship between the defect concentration or density and the induced strain. Note however that this assumption is valid only if interactions between elastic strain fields are limited, which might not be entirely justified for high defect concentrations, but which yet proves to be reasonable considering the results presented below. For point defects, the elastic strain is proportional to their so-called relaxation volume [54, 67, 68]. For dislocation loops, not only is it affected by the loop density, but it is also dependent on the size of the loops $[69,70]$. However, the characteristic features of the dislocation loops are not completely known in the current work in terms of size, geometry, etc., because they are often significantly entangled. Moreover, as stated earlier, there is no simple way to detect the loop character of a dislocation in an automated way. We therefore rely on the Burgers vector and visualization of dislocations to determine the loop character: $1 / 3<111>$ are (Frank) loops, but for perfect dislocations $1 / 2<110\rangle$, as stated above, we are unable to distinguish between loops and lines. Henceforth, for the sake of simplicity, we assumed that the elastic strain induced by dislocations is simply proportional to their density. Finally, the total elastic strain, $\varepsilon_{d e f}$, reads:

$$
\varepsilon_{d e f}=\frac{1}{3}\left[C_{V} \frac{V_{V}^{r e l}}{\Omega}+C_{I} \frac{V_{I}^{r e l}}{\Omega}+\left(S_{F} \rho_{F}+S_{p} \rho_{p}\right)\right]
$$

where $C_{V}$ and $C_{I}$ are concentrations of vacancies and interstitials, respectively, $V_{V}^{r e l}$ and $V_{I}^{r e l}$ are the relaxation volumes expressed in atomic volume unit $\Omega, \rho_{F}$ is the density of the Frank loops and $\rho_{p}$ is the density of perfect dislocations; the terms $S_{F}$ and $S_{p}$, which have the dimension of a surface, are the proportionality factors between concentration of Frank loops and perfect dislocations, and the corresponding strain, respectively.

It should be noticed that the sign of the relaxation volume of $U$ vacancy-type defects is still the object of debate in the literature [71, 72]. In the present study, we performed a pressure relaxation (MD) simulation to have direct access to this value. We obtained an average $0.6 \Omega$ value for $V_{V}^{r e l}$, where the average assumes an equiprobable distribution of single $\mathrm{U}$ vacancies, $\mathrm{U}$ divacancies and Schottky defects $(2 \mathrm{U}+\mathrm{O})$. We therefore performed fittings imposing a positive sign for $V_{V}^{r e l}$. The fitting of the strain kinetics with Eq.8, shown in Fig.5, was performed using a non-linear least squares optimization algorithm by minimizing the root-mean-square deviations (RMSD). Overall, the elastic strain kinetics is well fitted using Eq.8, demonstrating that the elastic strain determined from the MD simulations can be straightforwardly connected to the defect densities. Table I reports the values of the fitting parameters of Eq.8. It can be noticed that the value of $V_{V}^{r e l}$ was kept free during the fitting in order to allow for deviations from the simple equiprobable defect averaging.

$S_{F}$ is found to be $75 \AA^{2}$ while $S_{p}$ is $10 \AA^{2}$. These values demonstrate that the contribution of Frank loops to the overall strain level is around 8 times larger than that of perfect loops. 
This finding was expected since Frank loops, in contrast to perfect loops, have a nonvanishing average Burgers vector of $1 / 3<111>$. This difference implies that, while Frank loops essentially generate homogeneous (elastic) strain, perfect loops principally produce local (i.e., heterogeneous) strain. The same reasoning holds for perfect lines, where a random spatial distribution of dislocations or their arrangement into dipoles yield a vanishing average Burgers vector. Therefore, because of their limited contribution to the overall strain level, the inability to distinguish perfect dislocation loops from perfect dislocation lines does not have any measurable consequence. We show below that the situation is far different for the disorder.

Let us now consider the disorder, both derived from XRD and RBS/C. In a first step, similarly to what was done for the elastic strain, we assumed a linear relationship between defect densities and the induced disorder. Contrarily to the strain, vacancies do not contribute significantly to the disorder; their role was therefore neglected in the following. Fig.6 presents the corresponding fitting of the RBS/C disordering kinetics averaged over the three directions. It can be readily observed that this model provides a good description of the disordering process up to $0.4 \mathrm{dpU}$, which corresponds to the fluence at which the density of perfect dislocations reaches a maximum. Above $0.4 \mathrm{dpU}$, the model fails to describe the disorder, the most likely reason being that perfect loops and lines contribute significantly, but differently, to disorder, as opposed to their contribution to the strain. Being able to distinguish between perfect dislocation loops and lines is therefore crucial to get an accurate, quantitative description of the disordering process.

One solution to cope with this problem lies in the introduction of an ad hoc perfect line density, $\rho_{p}^{\text {line }}$, as a parameter that can be fitted and from which the perfect loop density, $\rho_{p}^{\text {loop }}$, can be derived, following the relationship: as $\rho_{p}^{\text {loop }}=\rho_{p}-\rho_{p}^{\text {line }}$. Based on the fit presented in Fig.6, we can safely assume that $\rho_{p}^{\text {line }}$ is not significant until around 0.4 dpU, i.e. where the fit quality deteriorates, and $\rho_{p}^{\text {line }}$ reaches a saturation level in the final line stage.

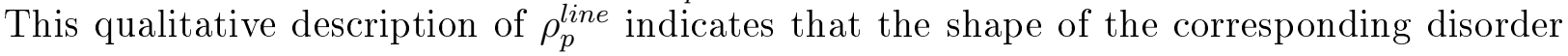
component should feature a high incubation time most likely followed by a sigmoidal growth spanning from 0.4 to $1.5 \mathrm{dpU}$ range, and a height determined by the final dislocation density, i.e. $0.26 \times 10^{17} \mathrm{~m}^{-2}$. The total disorder, $f_{d}$, can finally be represented by:

$$
f_{d}=r_{I} C_{I}+r_{F} \rho_{F}+r_{p}^{\text {loop }} \rho_{p}^{\text {loop }}+r_{p}^{\text {line }} \rho_{p}^{\text {line }}
$$

where $r_{I}$ is the disorder constant for the interstitials, and $r_{F}, r_{p}^{\text {loop }}$ and $r_{p}^{\text {line }}$ are the disorder constants for the Frank loops, perfect loops and perfect lines, respectively, with a $m^{2}$ unit.

Fig.7 shows the fits of the disordering kinetics obtained from (a) RBS/C and (b) XRD simulations using Eq.9. By comparing Fig.6 and Fig.7 (a), we observe that the quality of the fit in the high dpU region $(>0.4 \mathrm{dpU})$ is significantly improved after separating the contributions of perfect loops and lines. The decrease in disorder observed after $0.4 \mathrm{dpU}$ can therefore be ascribed to the reduction of the loop density at the expense of the perfect lines. The disorder constants are given in Table II. A feature worth putting forward from these constants is the different sensitivity of the two techniques, in terms of estimated disorder, to the nature of the dislocation loops. While $\mathrm{RBS} / \mathrm{C}$ is more sensitive to perfect loops, with a $r_{F} / r_{\text {loop }}^{p}$ ratio of 0.8 , the XRD phenomenon is more affected by Frank loops, with a 
corresponding ratio of 1.4. The reason for this difference is not entirely clear. We can note however that none of the RBS/C-derived and XRD-derived disorder values used here are absolute. As briefly above-mentioned, it is known that the backscattering yield depends on the probing-ion energy, and this dependence varies with the defect nature. Similarly, the Debye-Waller factor in XRD is a complex function of the scattering vector $Q$, and defects of different nature exhibit specific $Q$ dependencies. A careful inspection of both disorder parameters may hence allow to get very detailed and complementary information about the nature of the defects present in the MD cells but also in actual samples.

Examination of the constants in Table II provides another important result: dislocation lines have the largest influence on the disorder, as compared to the other defects, including perfect dislocation loops. This finding illustrates the complementarity between strain and disorder for the evaluation of the level of damage in irradiated materials. Indeed, because of the very nature of these two types of defects, namely perfect dislocation loops and lines, their effect on the disorder and on the strain is different. Dislocation loops are quasi 0dimensional defects, so their strain fields have a finite spatial extension and the long-rang order (LRO) of the crystal is weakly affected [73]. On the contrary, dislocation lines are 1-dimensional defects with a diverging $\ln (r)$ term in the displacement field that significantly alters the LRO of the crystal. The fact the strain kinetic reaches its maximum earlier (in terms of $\mathrm{dpU}$ ) than the disorder can be entirely explained by this different sensitivity to the defect type. As a final comment, it is worth mentioning that the number of damage metrics, two in the current work, namely the strain and the disorder, defines the number of defect transformations that can be studied. If there were an additional type of defect, say stacking faults or precipitates, then another metric would be needed to monitor the defect evolution with time. In the present case, we limited the use of XRD data to the intensity and the strain. However, additional parameters, such as the reflection broadening also conveys information regarding the defect structure. This will the topic of a forthcoming paper. Another solution to deal with a higher number of defect types would be to implement additional techniques, such as Raman [74] or positron annihilation spectroscopy [75] for which it is possible to generate computed signals.

\section{Conclusions}

Summarizing, we generated experimental-like signals of RBS/C and XRD techniques using the same MD dataset produced with the Frenkel pair accumulation method. We then computed disordering kinetics, with disorder and strain parameters to monitor the damage accumulating in the MD cells. We demonstrated that, on the one hand, both techniques can be used interchangeably to quantify the disorder in defective cells and in actual materials. On the other hand, because they have a different sensitivity on the probed defects, their combination can provide complementary information about the defect nature. Despite discrepancies in terms of absolute values of fluence range and levels of strain and disorder, on a relative scale, the computed strain and disordering kinetics agree well with those determined from actual experiments, which demonstrates the validity of the integrated approach. Additional work is in progress with other materials to provide further support and improve the methodology. Nonetheless, the results provided in this paper can be transposed to materials exhibiting the same disordering behaviour. 


\section{Acknowledgments}

X. Jin is grateful to SIMME Doctoral School (Université de Limoges) and PHENIICS Doctoral School (Université Paris-Saclay) for the financial support of his PhD research work. Frédérico Garrido and Lionel Thomé are acknowledged for fruitful discussions. Part of this research work has been funded within the NEEDS-CNRS program.

\section{Competing interests}

The authors declare no conflict of interest. 


\section{Figures}

a
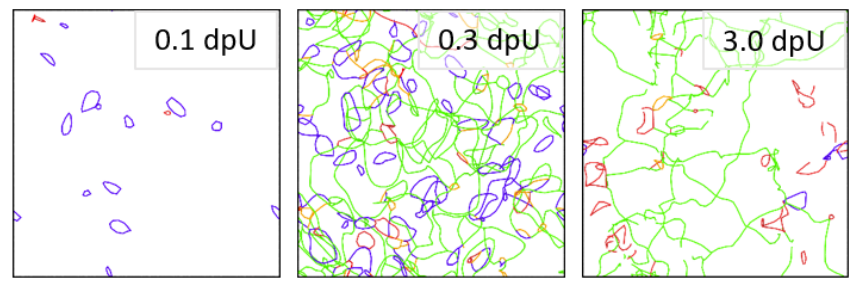

C

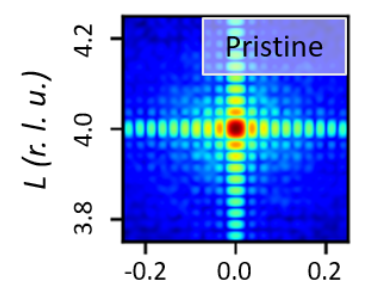

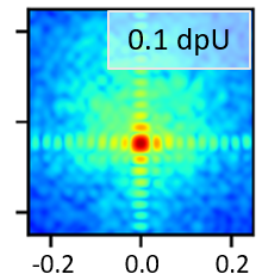

$H$ (r. I. u.)
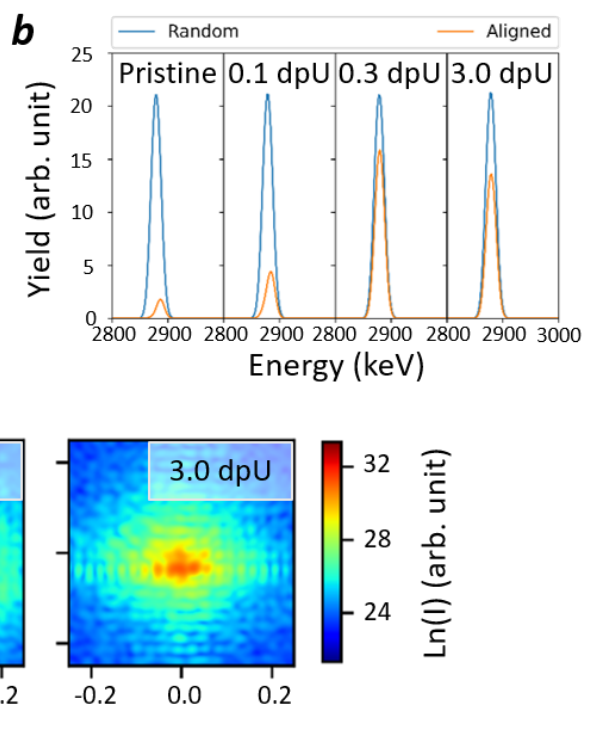

Figure 1: Characteristic microstructures obtained by MD simulations and corresponding $\mathrm{RBS} / \mathrm{C}$ and $\mathrm{XRD}$ signals generated from the $\mathrm{UO}_{2} \mathrm{MD}$ cells. a Snapshots of $\mathrm{UO}_{2} \mathrm{MD}$ cells analyzed by the DXA method with different damage levels: $0.1 \mathrm{dpU}$, $0.3 \mathrm{dpU}$ and $3.0 \mathrm{dpU}$. Only dislocations including Frank loops (blue), perfect dislocations (green) and Shockley partial dislocations (orange) in the cells are visualized. (Other types of dislocations including stair-rod dislocations and ill-defined dislocations are represented by red lines.) b RBS/C spectra of $3.085 \mathrm{MeV} \mathrm{He}$ on the [001]-oriented $\mathrm{UO}_{2} \mathrm{MD}$ cells presented in a. Blue lines represent random spectra, and orange lines represent aligned spectra. c RSMs calculated in the vicinity of the [004] Bragg reflection of the $\mathrm{UO}_{2} \mathrm{MD}$ cells presented in a. Units of $H$ and $L$ are reciprocal lattice units (r.l.u) along the $\mathrm{x}$ and $\mathrm{z}$ directions, respectively. Intensities indicated by the color bar are represented on a logarithmic scale. 


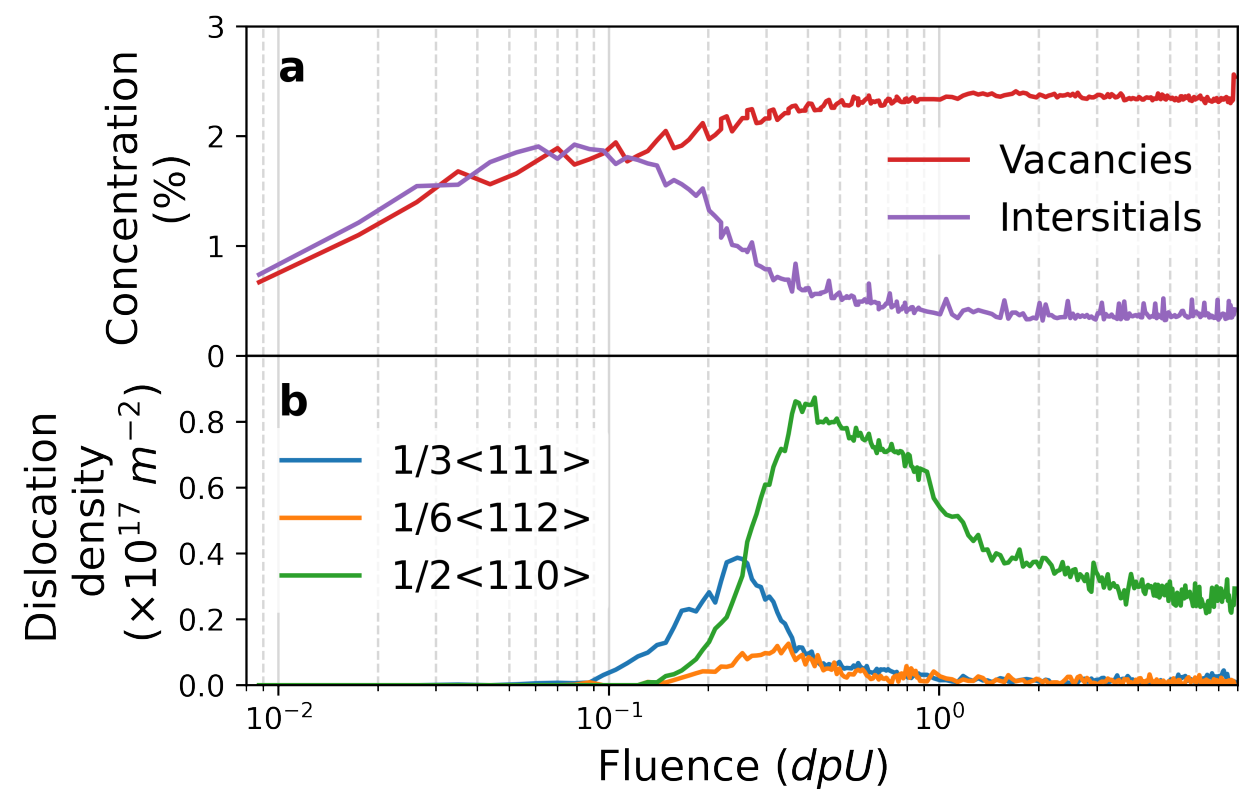

Figure 2: Defect quantities as a function of fluence (dpU). a Concentrations of vacancies and of interstitials, $\mathbf{b}$ Densities of $1 / 3<111>$ Frank loops, of $1 / 6<112>$ Shockley partial dislocations and of $1 / 2<110>$ perfect dislocations. 

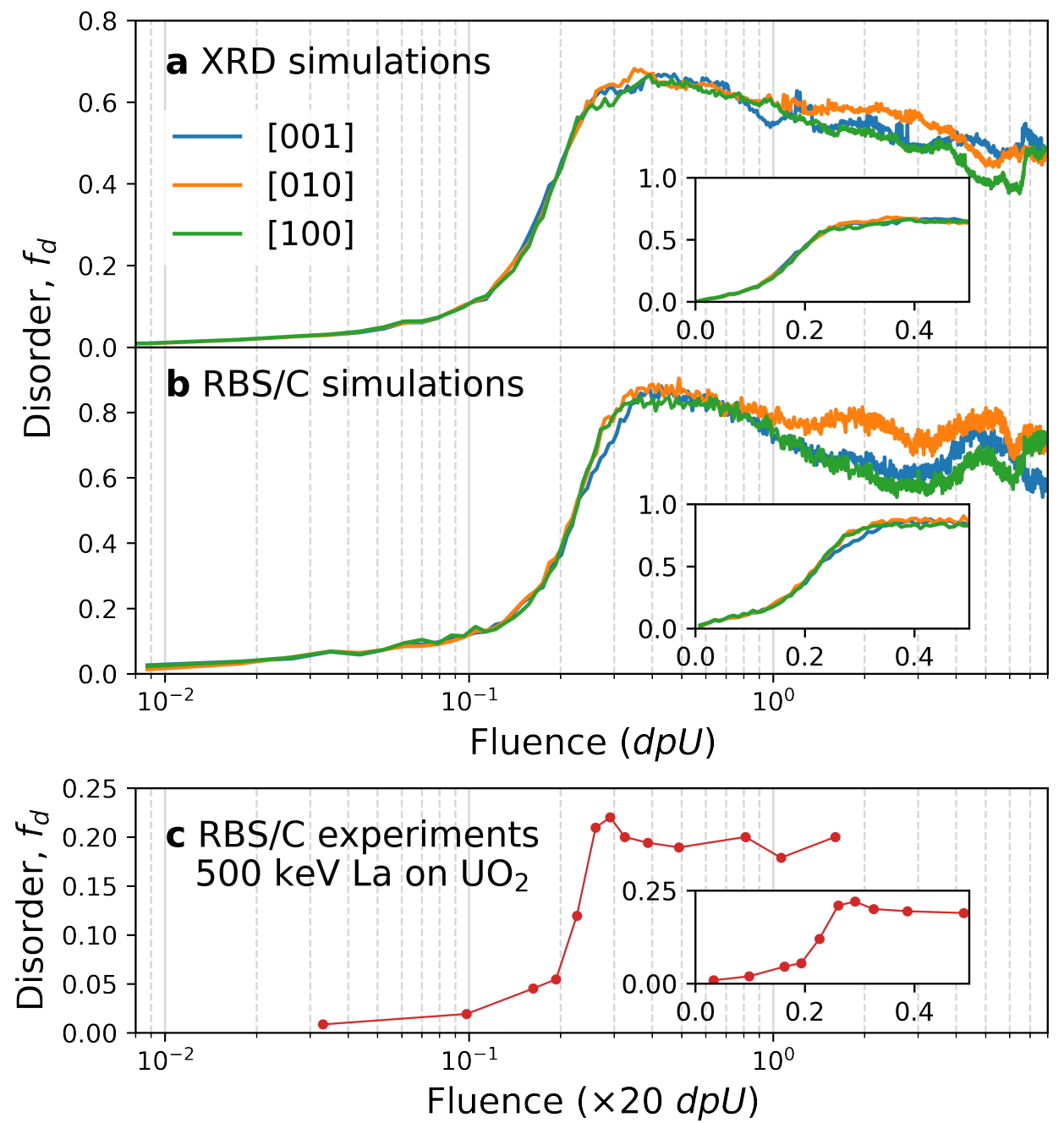

Figure 3: Disordering kinetics obtained from: a The XRD simulations on the $\mathrm{UO}_{2}$ MD cells, $\mathbf{b}$ the RBS/C simulations on the $\mathrm{UO}_{2} \mathrm{MD}$ cells, $\mathbf{c}$ RBS/C experiments on $\mathrm{UO}_{2}$ crystals irradiated with $500 \mathrm{keV}$ La ions. In the simulations, the MD cells were characterized along three major directions: [001] (blue lines), [010] (orange lines) and [100] (green lines). Insets show part of the kinetics using a linear scale for the $\mathrm{x}$ axis. 


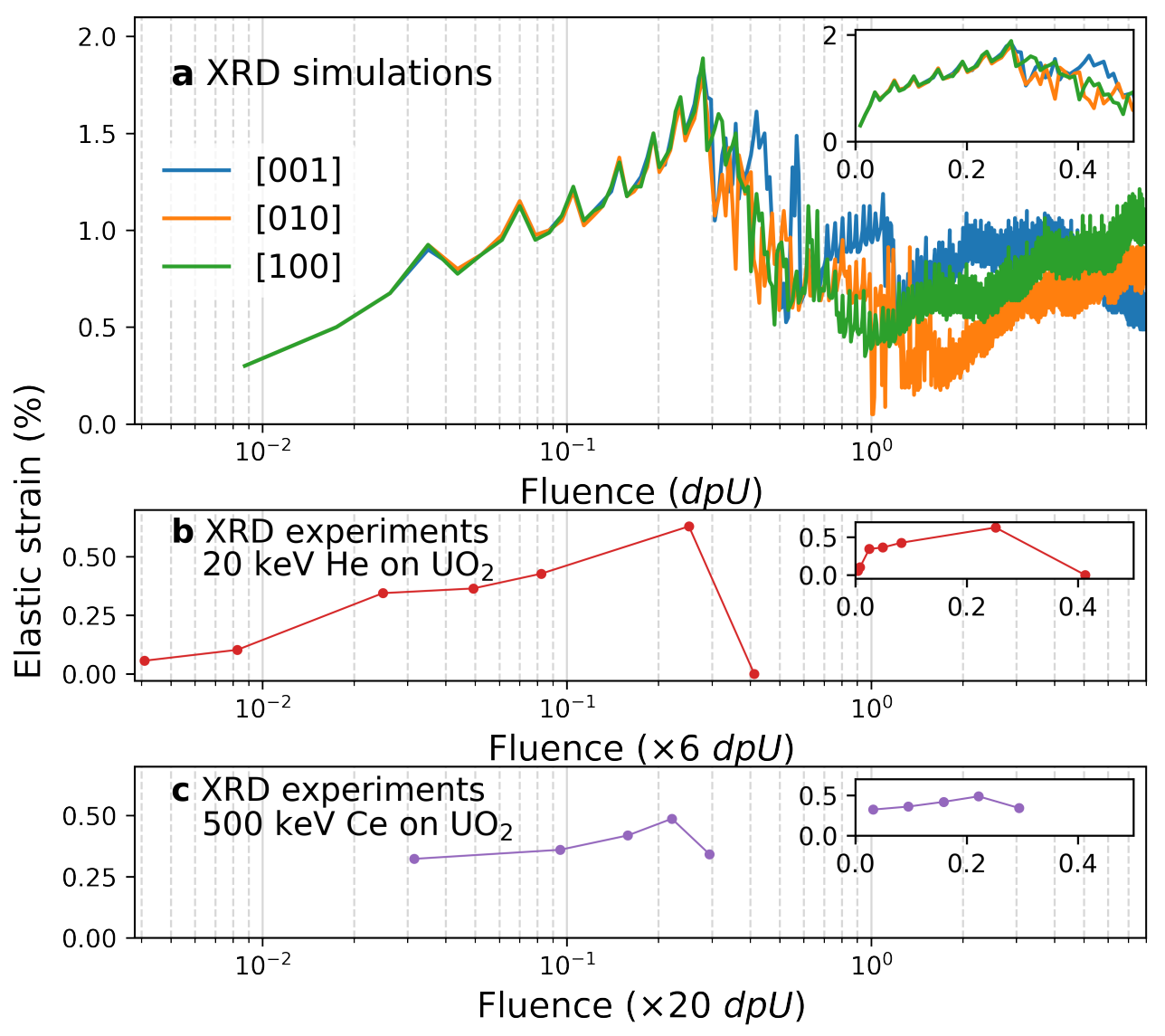

Figure 4: Elastic strain kinetics obtained from: a The XRD simulations on the $\mathrm{UO}_{2}$ MD cells, b XRD experiments of $20 \mathrm{keV} \mathrm{He}$ on $\mathrm{UO}_{2}$ crystals, c XRD experiments of 500 $\mathrm{keV} \mathrm{Ce}$ on $\mathrm{UO}_{2}$ samples. In the simulations, the MD cells were characterized along three major directions: [001] (blue lines), [010] (orange lines) and [100] (green lines). Samples in experiments were characterized along $<001\rangle$ directions. Insets show part of the kinetics using a linear scale for the $\mathrm{x}$ axis. 


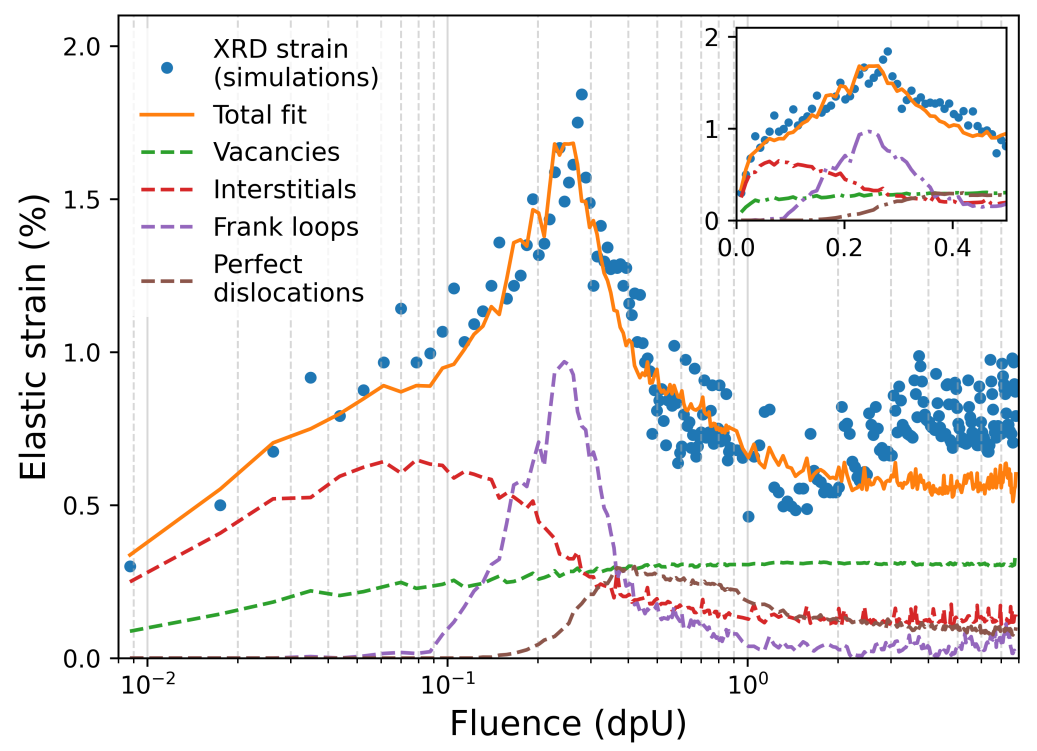

Figure 5: Fit of the elastic strain kinetics. The blue dots represent the elastic strain computed from the XRD simulations and averaged over the three $<001>$ directions. The solid line represents the total fit by combining contributions of the different defects (dashed lines). The RMSD value equals to $1.7 \times 10^{-1}$. The inset shows part of the kinetics using a linear scale for the $\mathrm{x}$ axis. (In order to avoid a dense overlap, only one value in every five simulation results is shown when the fluence is larger than $1.0 \mathrm{dpU}$.)

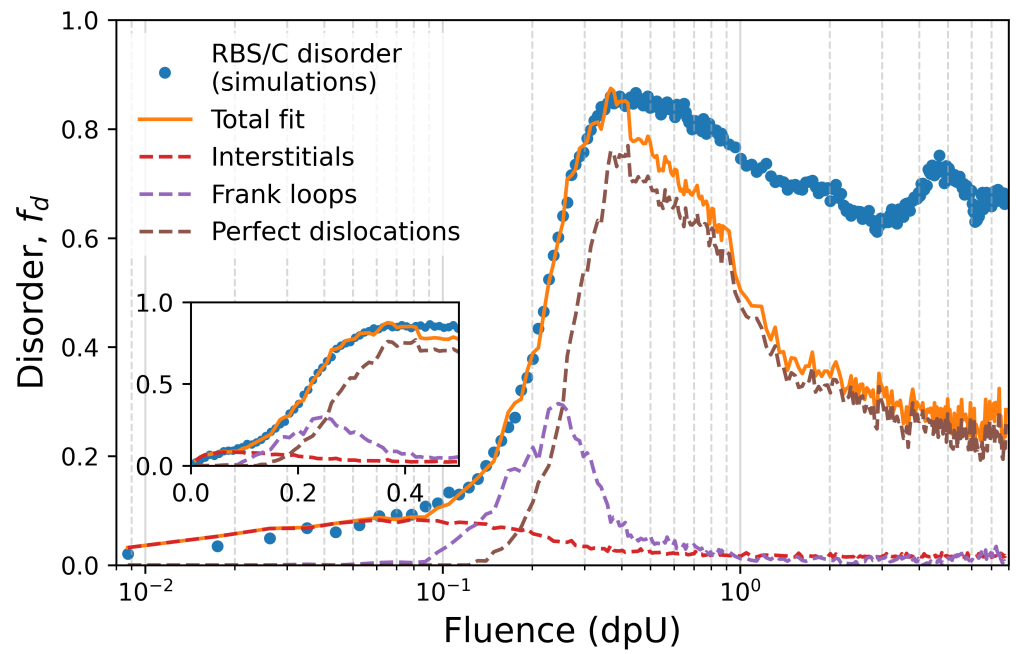

Figure 6: Fit of the disordering kinetics assuming proportionality between defect quantities and the induced disorder. The blue dots represent the disorder computed from the RBS/C simulations and averaged over the three $<001>$ directions. The solid line represents the total fit by combining the contributions of the different defects (dashed lines). The inset shows part of the kinetics using a linear scale for the $\mathrm{x}$ axis. (In order to avoid a dense overlap, only one value in every five simulation results is shown when the fluence is larger than $1.0 \mathrm{dpU}$.) 

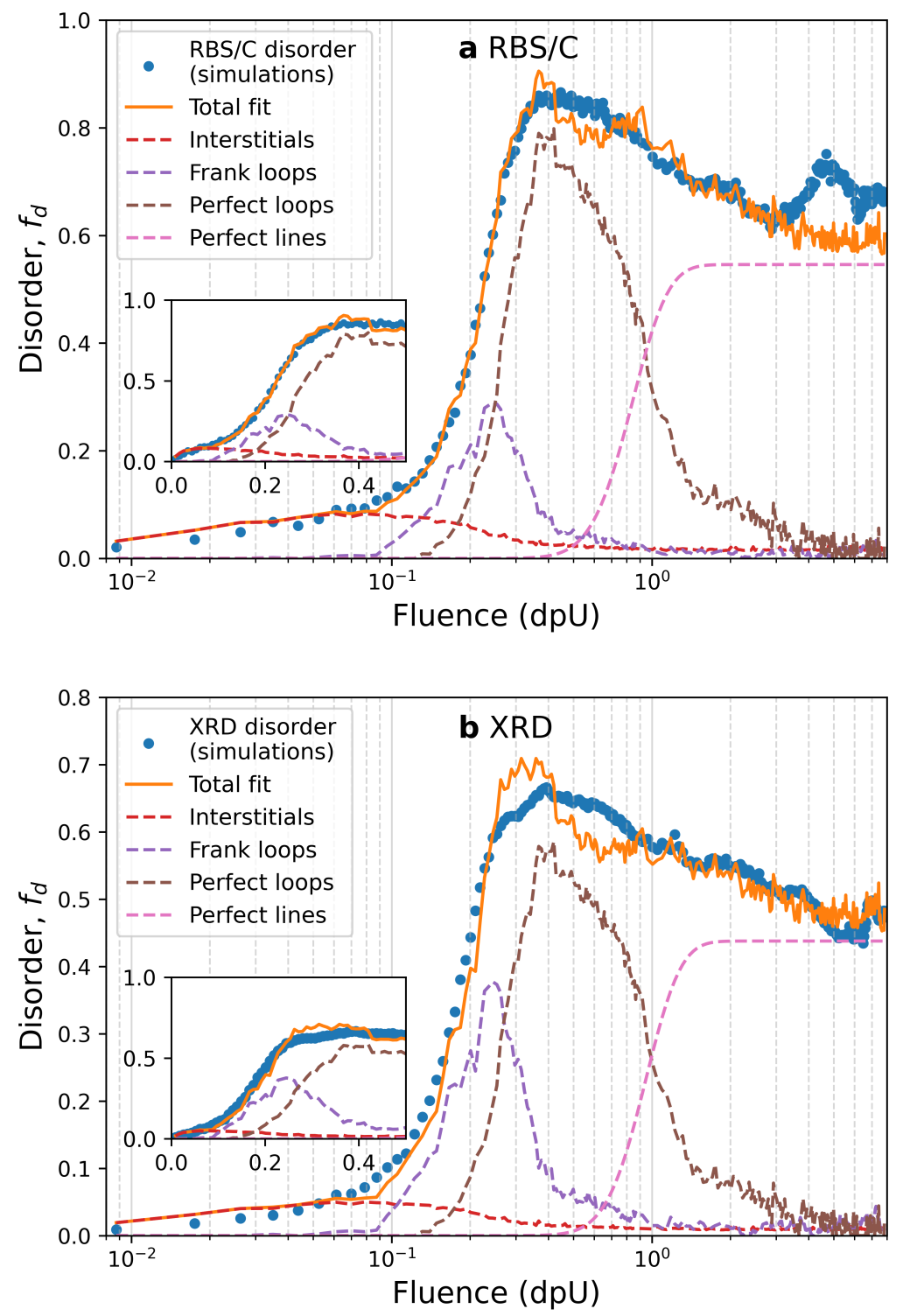

Figure 7: Fits of the disordering kinetics assuming proportionality between defect quantities and the induced disorder and separating the perfect loop and line contributions. The blue dots represent the averaged disorder computed from a RBS/C simulations $\left(\mathrm{RMSD}=6.5 \times 10^{-2}\right)$ and $\mathbf{b} \mathrm{XRD}$ simulations $\left(\mathrm{RMSD}=3.2 \times 10^{-2}\right)$. Insets show part of the kinetics using a linear scale for the $\mathrm{x}$ axis. (In order to avoid dense overlap, only one value in every five simulation results is shown when the fluence is larger than 1.0 dpU.) 


\section{Tables}

Table I: Relaxation volumes, $V^{\text {rel }}$, of point defects and strain constants, $S$, of dislocations. The errors are obtained from the non-linear least squares fitting algorithm.

\begin{tabular}{cccc}
\hline \hline$V_{V}^{\text {rel }}(\Omega)$ & $V_{I}^{\text {rel }}(\Omega)$ & $S_{F}\left(\AA^{2}\right)$ & $S_{p}\left(\AA^{2}\right)$ \\
\hline $0.4 \pm 0.1$ & $1.0 \pm 0.1$ & $75.1 \pm 3.5$ & $10.3 \pm 2.4$ \\
\hline \hline
\end{tabular}

Table II: Disorder constants, $r$, for defects used in the fits. a RBS/C disordering kinetics, $\mathbf{b}$ XRD disordering kinetics. The errors are obtained from the non-linear least squares fitting algorithm.

\begin{tabular}{c|cccc}
\hline \hline & $r_{I}$ & $r_{F}\left(\times 10^{-17} m^{2}\right)$ & $r_{p}^{\text {loop }}\left(\times 10^{-17} m^{2}\right)$ & $r_{p}^{\text {line }}\left(\times 10^{-17} m^{2}\right)$ \\
\hline RBS $/ \mathrm{C}$ & $4.31 \pm 0.96$ & $0.75 \pm 0.07$ & $0.92 \pm 0.02$ & $2.10 \pm 0.08$ \\
XRD & $2.60 \pm 0.47$ & $0.97 \pm 0.03$ & $0.67 \pm 0.08$ & $1.71 \pm 0.05$ \\
\hline \hline
\end{tabular}

\section{References}

[1] U. Amaldi, S. Braccini, Present challenges in hadrontherapy techniques, Eur. Phys. J. Plus 126 (2011) 1-15.

[2] ATLAS Collaboration, CMS Collaboration, Combined Measurement of the Higgs Boson Mass in $p p$ Collisions at $\sqrt{s}=7$ and $8 \mathrm{TeV}$ with the ATLAS and CMS Experiments, Phys. Rev. Lett. 114 (2015) 1-33.

[3] M. Durante, A. Golubev, W. Y. Park, C. Trautmann, Applied nuclear physics at the new high-energy particle accelerator facilities, Phys. Rep. 800 (2019) 1-37.

[4] E. M. Bringa, S. O. Kucheyev, M. J. Loeffler, R. A. Baragiola, A. G. G. M. Tielens, Z. R. Dai, G. Graham, S. Bajt, J. P. Bradley, C. A. Dukes, T. E. Felter, D. F. Torres, W. van Breugel, Energetic Processing of Interstellar Silicate Grains by Cosmic Rays, ApJ 662 (2007) 372-378.

[5] E. Chason, S. T. Picraux, J. M. Poate, J. O. Borland, M. I. Current, T. Diaz De La Rubia, D. J. Eaglesham, O. W. Holland, M. E. Law, C. W. Magee, J. W. Mayer, J. Melngailis, A. F. Tasch, Ion beams in silicon processing and characterization, J. Appl. Phys. 81 (1997) 6513-6561.

[6] L. Rubin, J. Poate, Ion implantation in silicon technology, The Industrial Physicist 9 (2003) 12-15.

[7] E. Nogales, P. Hidalgo, K. Lorenz, B. Méndez, J. Piqueras, E. Alves, Cathodoluminescence of rare earth implanted $\mathrm{Ga}_{2} \mathrm{O}_{3}$ and $\mathrm{GeO}_{2}$ nanostructures, Nanotechnology 22 (2011). 
[8] N. Theodoropoulou, A. F. Hebard, M. E. Overberg, C. R. Abernathy, S. J. Pearton, S. N. Chu, R. G. Wilson, Unconventional Carrier-Mediated Ferromagnetism above Room Temperature in Ion-Implanted (Ga, Mn)P:C, Phys. Rev. Lett. 89 (2002) 3-6.

[9] N. Ocelic, R. Hillenbrand, Subwavelength-scale tailoring of surface phonon polaritons by focused ion-beam implantation, Nat. Mater. 3 (2004) 606-609.

[10] B. Peng, M. Locascio, P. Zapol, S. Li, S. L. Mielke, G. C. Schatz, H. D. Espinosa, Measurements of near-ultimate strength for multiwalled carbon nanotubes and irradiationinduced crosslinking improvements, Nat. Nanotechnol. 3 (2008) 626-631.

[11] C. Gómez-Navarro, P. J. De Pablo, J. Gómez-Herrero, B. Biel, F. J. Garcia-Vidal, A. Rubio, F. Flores, Tuning the conductance of single-walled carbon nanotubes by ion irradiation in the Anderson localization regime, Nat. Mater. 4 (2005) 534-539.

[12] S. Talapatra, P. G. Ganesan, T. Kim, R. Vajtai, M. Huang, M. Shima, G. Ramanath, D. Srivastava, S. C. Deevi, P. M. Ajayan, Irradiation-induced magnetism in carbon nanostructures, Phys. Rev. Lett. 95 (2005) 15-18.

[13] J. H. Warner, M. H. Rümmeli, L. Ge, T. Gemming, B. Montanari, N. M. Harrison, B. Büchner, G. A. D. Briggs, Structural transformations in graphene studied with high spatial and temporal resolution, Nat. Nanotechnol. 4 (2009) 500-504.

[14] A. V. Krasheninnikov, K. Nordlund, Ion and electron irradiation-induced effects in nanostructured materials, J. Appl. Phys. 107 (2010).

[15] O. Ochedowski, O. Osmani, M. Schade, B. K. Bussmann, B. Ban-Detat, H. Lebius, M. Schleberger, Graphitic nanostripes in silicon carbide surfaces created by swift heavy ion irradiation, Nat. Commun. 5 (2014) 1-8.

[16] G. S. Was, Challenges to the use of ion irradiation for emulating reactor irradiation, J. Mater. Res. 30 (2015) 1158-1182.

[17] S. J. Zinkle, L. L. Snead, Opportunities and limitations for ion beams in radiation effects studies: Bridging critical gaps between charged particle and neutron irradiations, Scr. Mater. 143 (2018) 154-160.

[18] D. Stork, R. Heidinger, T. Muroga, S. J. Zinkle, A. Moeslang, M. Porton, J. L. Boutard, S. Gonzalez, A. Ibarra, Towards a programme of testing and qualification for structural and plasma-facing materials in 'fusion neutron' environments, Nucl. Fusion 57 (2017).

[19] M. T. Robinson, Basic physics of radiation damage production, J. Nucl. Mater. 216 (1994) 1-28.

[20] W. Wesch, E. Wendler (Eds.), Ion Beam Modification of Solids: Ion-Solid Interaction and Radiation Damage, volume 61, Springer, 2016.

[21] M. Victoria, N. Baluc, C. Bailat, Y. Dai, M. I. Luppo, R. Schaublin, B. N. Singh, Microstructure and associated tensile properties of irradiated fcc and bcc metals, J. Nucl. Mater. 276 (2000) 114-122. 
[22] C. Ronchi, M. Sheindlin, D. Staicu, M. Kinoshita, Effect of burn-up on the thermal conductivity of uranium dioxide up to $100.000 \mathrm{MWdt}^{-1}$, J. Nucl. Mater. 327 (2004) $58-76$.

[23] W. J. Weber, Radiation-induced amorphization in $\mathrm{Ca}_{2} \mathrm{Nd}_{8}\left(\mathrm{SiO}_{4}\right)_{6} \mathrm{O}_{2}$, Radiat. Eff. 77 (1983) 295-308.

[24] I. J. Hastings, M. J. Notley, D. H. Rose, Irradiation-induced volume changes in commercial $\mathrm{UO}_{2}$ fuel: Comparison with model prediction, J. Nucl. Mater. 75 (1978) 301-303.

[25] W. Jiang, C. M. Wang, W. J. Weber, M. H. Engelhard, L. V. Saraf, Direct determination of volume changes in ion-beam-irradiated SiC, J. Appl. Phys. 95 (2004) 4687-4690.

[26] Y. Zhang, A. Debelle, A. Boulle, P. Kluth, F. Tuomisto, Advanced techniques for characterization of ion beam modified materials, Curr Opin Solid State Mater Sci 19 (2015) 19-28.

[27] C. S. Becquart, C. Domain, Modeling microstructure and irradiation effects, Metall Mater Trans A Phys Metall Mater Sci 42 (2011) 852-870.

[28] K. Nordlund, Historical review of computer simulation of radiation effects in materials, J. Nucl. Mater. (2019) 273-295.

[29] T. Wiss, Radiation effects in $\mathrm{UO}_{2}$, volume 2, Elsevier Inc., 2012.

[30] J. P. Crocombette, A. Chartier, W. J. Weber, Atomistic simulation of amorphization thermokinetics in lanthanum pyrozirconate, Appl. Phys. Lett. 88 (2006) 1-3.

[31] D. S. Aidhy, P. C. Millett, T. Desai, D. Wolf, S. R. Phillpot, Kinetically evolving irradiation-induced point defect clusters in $\mathrm{UO}_{2}$ by molecular dynamics simulation, Phys. Rev. B Condens. Matter Mater. Phys. 80 (2009) 1-9.

[32] D. S. Aidhy, C. Lu, K. Jin, H. Bei, Y. Zhang, L. Wang, W. J. Weber, Point defect evolution in $\mathrm{Ni}, \mathrm{NiFe}$ and $\mathrm{NiCr}$ alloys from atomistic simulations and irradiation experiments, Acta Mater. 99 (2015) 69-76.

[33] A. Chartier, C. Onofri, L. Van Brutzel, C. Sabathier, O. Dorosh, J. Jagielski, Early stages of irradiation induced dislocations in urania, Appl. Phys. Lett. 109 (2016).

[34] A. Debelle, A. Boulle, A. Chartier, F. Gao, W. J. Weber, Interplay between atomic disorder, lattice swelling, and defect energy in ion-irradiation-induced amorphization of SiC, Phys. Rev. B Condens. Matter Mater. Phys. 90 (2014) 13-17.

[35] A. Chartier, M. C. Marinica, Rearrangement of interstitial defects in alpha-Fe under extreme condition, Acta Mater. 180 (2019) 141-148.

[36] J. F. Ziegler, SRIM, Available at http://www.srim.org/.

[37] J. Soullard, High voltage electron microscope observations of $\mathrm{UO}_{2}$, J. Nucl. Mater. 135 (1985) 190-196. 
[38] R. E. Stoller, M. B. Toloczko, G. S. Was, A. G. Certain, S. Dwaraknath, F. A. Garner, On the use of SRIM for computing radiation damage exposure, Nucl. Instrum. Methods Phys. Res., B 310 (2013) 75-80.

[39] L. Van Brutzel, J. M. Delaye, D. Ghaleb, M. Rarivomanantsoa, Molecular dynamics studies of displacement cascades in the uranium dioxide matrix, Philos Mag (Abingdon) 83 (2003) 4083-4101.

[40] A. Stukowski, Visualization and analysis of atomistic simulation data with OVITO-the Open Visualization Tool, Model. Simul. Mat. Sci. Eng. 18 (2010).

[41] A. Stukowski, Structure identification methods for atomistic simulations of crystalline materials, Model. Simul. Mat. Sci. Eng. 20 (2012) 1-15.

[42] A. Stukowski, K. Albe, Extracting dislocations and non-dislocation crystal defects from atomistic simulation data, Model. Simul. Mat. Sci. Eng. 18 (2010).

[43] A. Stukowski, V. V. Bulatov, A. Arsenlis, Automated identification and indexing of dislocations in crystal interfaces, Model. Simul. Mat. Sci. Eng. 20 (2012).

[44] S. Zhang, K. Nordlund, F. Djurabekova, Y. Zhang, G. Velisa, T. S. Wang, Simulation of Rutherford backscattering spectrometry from arbitrary atom structures, Phys. Rev. E 94 (2016) 1-12.

[45] X. Jin, J.-P. Crocombette, F. Djurabekova, S. Zhang, K. Nordlund, F. Garrido, A. Debelle, New developments in the simulation of Rutherford backscattering spectrometry in channeling mode using arbitrary atom structures, Model. Simul. Mat. Sci. Eng. 28 (2020) 1-23.

[46] M. Robinson, I. Torrens, Computer simulation of atomic-displacement cascades in solids in the binary-collision approximation, Phys. Rev. B 9 (1974) 5008-5024.

[47] F. Garrido, L. Nowicki, L. Thomé, Channeling investigation of the crystalline structure of U4 O9-y, Phys. Rev. B Condens. Matter Mater. Phys. 74 (2006) 1-13.

[48] B. Warren, X-Ray Diffraction, Addison-Wesley, 1969.

[49] J. Channagiri, A. Boulle, A. Debelle, Diffuse X-ray scattering from ion-irradiated materials: A parallel-computing approach, J. Appl. Crystallogr. 48 (2015) 252-261.

[50] A. Boulle, A. Chartier, J.-P. Crocombette, T. Jourdan, S. Pellegrino, A. Debelle, Strain and damage build-up in irradiated crystals: Coupling X-ray diffraction with numerical simulations, Nucl. Instrum. Methods Phys. Res., B 458 (2019) 143-150.

[51] D. Hull, D. J. Bacon, Introduction to dislocations, fifth ed., Butterworth-Heinemann, 2011.

[52] C. Onofri, C. Sabathier, H. Palancher, G. Carlot, S. Miro, Y. Serruys, L. Desgranges, M. Legros, Evolution of extended defects in polycrystalline $\mathrm{UO}_{2}$ under heavy ion irradiation: Combined TEM, XRD and Raman study, Nucl. Instrum. Methods Phys. Res., B 374 (2016) 51-57. 
[53] C. Onofri, M. Legros, J. Léchelle, H. Palancher, C. Baumier, C. Bachelet, C. Sabathier, Full characterization of dislocations in ion-irradiated polycrystalline UO2, J. Nucl. Mater. 494 (2017) 252-259.

[54] A. Debelle, J.-P. Crocombette, A. Boulle, A. Chartier, T. Jourdan, S. Pellegrino, D. Bachiller-Perea, D. Carpentier, J. Channagiri, T. H. Nguyen, F. Garrido, L. Thomé, Lattice strain in irradiated materials unveils a prevalent defect evolution mechanism, Phys. Rev. Mater. 2 (2018) 1-8.

[55] A. Boulle, A. Debelle, Statistical Nature of Atomic Disorder in Irradiated Crystals, Phys. Rev. Lett. 116 (2016) 1-5.

[56] H. Matzke, A. Turos, G. Linker, Polygonization of single crystals of the fluorite-type oxide $\mathrm{UO}_{2}$ due to high dose ion implantation, Nucl. Instrum. Methods Phys. Res., B 91 (1994) 294-300.

[57] K. Nogita, K. Une, Radiation-induced microstructural change in high burnup $\mathrm{UO}_{2}$ fuel pellets, Nucl. Instrum. Methods Phys. Res., B 91 (1994) 301-306.

[58] A. Debelle, J. P. Crocombette, A. Boulle, E. Martinez, B. P. Uberuaga, D. BachillerPerea, Y. Haddad, F. Garrido, L. Thomé, M. Béhar, How relative defect migration energies drive contrasting temperature-dependent microstructural evolution in irradiated ceramics, Phys. Rev. Mater. 2 (2018) 1-8.

[59] J. Rest, A model for the influence of microstructure, precipitate pinning and fission gas behavior on irradiation-induced recrystallization of nuclear fuels, J. Nucl. Mater. 326 (2004) 175-184.

[60] L. Van Brutzel, M. Rarivomanantsoa, D. Ghaleb, Displacement cascade initiated with the realistic energy of the recoil nucleus in UO2 matrix by molecular dynamics simulation, J. Nucl. Mater. 354 (2006) 28-35.

[61] J. P. Crocombette, L. Van Brutzel, D. Simeone, L. Luneville, Molecular dynamics simulations of high energy cascade in ordered alloys: Defect production and subcascade division, J. Nucl. Mater. 474 (2016) 134-142.

[62] S. Moll, L. Thomé, G. Sattonnay, A. Debelle, F. Garrido, L. Vincent, J. Jagielski, Multistep damage evolution process in cubic zirconia irradiated with $\mathrm{MeV}$ ions, J. Appl. Phys. 106 (2009) 2-11.

[63] A. Debelle, J. Channagiri, L. Thomé, B. Décamps, A. Boulle, S. Moll, F. Garrido, M. Behar, J. Jagielski, Comprehensive study of the effect of the irradiation temperature on the behavior of cubic zirconia, J. Appl. Phys. 115 (2014) 1-9.

[64] S. Moll, Y. Zhang, A. Debelle, L. Thomé, J. P. Crocombette, Z. Zihua, J. Jagielski, W. J. Weber, Damage processes in MgO irradiated with medium-energy heavy ions, Acta Mater. 88 (2015) 314-322.

[65] G. Velişa, K. Jin, Z. Fan, C. Lu, H. Bei, W. J. Weber, L. Wang, Y. Zhang, Multi-axial and multi-energy channeling study of disorder evolution in ion-irradiated nickel, J. Nucl. Mater. 525 (2019) 92-101. 
[66] K. Jin, H. Bei, Y. Zhang, Ion irradiation induced defect evolution in Ni and Ni-based FCC equiatomic binary alloys, J. Nucl. Mater. 471 (2016) 193-199.

[67] P. H. Dederichs, C. Lehmann, H. R. Schober, A. Scholz, R. Zeller, Lattice theory of point defects, J. Nucl. Mater. 70 (1978) 176-199.

[68] D. Bachiller-Perea, A. Debelle, L. Thomé, J. P. Crocombette, Study of the initial stages of defect generation in ion-irradiated $\mathrm{MgO}$ at elevated temperatures using highresolution X-ray diffraction, J. Mater. Sci. 51 (2016) 1456-1462.

[69] P. H. Dederichs, The theory of diffuse X-ray scattering and its application to the study of point defects and their clusters, J Phys F Met Phys 3 (1973) 471-496.

[70] M. W. Ullah, Y. Zhang, N. Sellami, A. Debelle, H. Bei, W. J. Weber, Evolution of irradiation-induced strain in an equiatomic NiFe alloy, Scr. Mater. 140 (2017) 35-39.

[71] A. Goyal, K. Mathew, R. G. Hennig, A. Chernatynskiy, C. R. Stanek, S. T. Murphy, D. A. Andersson, S. R. Phillpot, B. P. Uberuaga, The conundrum of relaxation volumes in first-principles calculations of charged defects in $\mathrm{UO}_{2}$, Appl. Sci. 9 (2019) 1-24.

[72] M. Freyss, T. Petit, J. P. Crocombette, Point defects in uranium dioxide: Ab initio pseudopotential approach in the generalized gradient approximation, J. Nucl. Mater. 347 (2005) 44-51.

[73] M. Krivoglaz, Theory of X-Ray and Thermal-Neutron Scattering by Real Crystals, Springer, 1969.

[74] G. Roma, Modeling the contribution of point defects to the Raman spectrum of crystalline materials, Model. Simul. Mat. Sci. Eng. 27 (2019).

[75] J. Wiktor, M. F. Barthe, G. Jomard, M. Torrent, M. Freyss, M. Bertolus, Coupled experimental and DFT $+\mathrm{U}$ investigation of positron lifetimes in UO2, Phys. Rev. B Condens. Matter Mater. Phys. 90 (2014) 1-11. 